\title{
LA OBJECIÓN DE CONCIENCIA DEL \\ PERSONALDE SALUD ANTE LA \\ VOLUNTAD ANTICIPADA: \\ REFLEXIONES PARA UNA MUERTE DIGNA
}

\begin{abstract}
AWARENESS OBJECTION AMONG HEALTH PERSONNEL IN THE FACE OF THE PATIENT'S ANTICIPATED WILL: REFLECTIONS ON A DIGNIFIED DEATH
\end{abstract}

\section{Autores:}

Dra. Elliug Osuna Suárez

Médica Cirujana y Lic. en Derecho, por la UNAM. Profesora de Asignatura B de la ENEO de la UNAM

Dra. Lasty Balseiro Almario

Profesora de Carrera Titular B de la ENEO de la UNAM. Adscrita a la Unidad de Investigación

Palabras clave: Adolescentes, autoel̈cacia, condón

Key words: Adolescents, self-eflicacy, condom 


\section{RESUMEN}

El presente ensayo tiene por objeto motivar en los profesionales de la salud a la reflexión sobre la Objeción de Conciencia ante la Voluntad Anticipada de los pacientes. Para ello, se han desarrollado lo siguientes apartados: en un primer lugar se analizan los conceptos de la Objeción de Conciencia y de Voluntad Anticipada, así como los antecedentes de la Objeción de Conciencia, los valores éticos derivados de la autonomía de la conciencia individual, los aspectos legales de la Objeción de Conciencia y la muerte digna. Con todos estos antecedentes, se analizan en un segundo momento, los derechos humanos fundamentales, la objeción y los conflictos, la toma de decisiones en la práctica médica y en la enfermería, especialmente en el contexto de los enfermos terminales, el estado de coma de los pacientes o un estado de sufrimiento irreversible. Finaliza este ensayo con las reflexiones entre la vida y la muerte y la posibilidad de intervenir con métodos y tecnologías avanzadas para prolongar o terminar la vida. En la práctica de la enfermería son necesarias estas reflexiones en apego irrestricto al respeto y la protección de los derechos de la dignidad humana de los pacientes.

Palabras clave. Conciencia, Objeción, Paciente, Personal de Salud, Voluntad.

\section{ABSTRACT}

The present study aims to motivate health personnel to reflecting on the Awareness Objection in the face of the patient's Anticipated Will. The issue is addressed analyzing, in a first moment, the concepts of Awareness Objection and

Anticipated Will, the background of Awareness Objection, the ethical values derived from the autonomy of the individual awareness, the legal aspects related to the Awareness Objection, and a dignified death. In a second moment, aspects such as the fundamental human rights, the objection and conflict, the decision making in medical and nursing practice - particularly within the contexts of terminal patients who, among other, could be in coma or an irreversible suffering state, are analyzed. The study concludes with reflections on life and death, and the possibility to intervene with advanced methods and technologies in order to prolong or terminate life. In the practice of nursing, all of these reflections are necessary under the consideration of strict respect and protection to the rights and dignity of the patients.

Key words: Awareness; Objection; Patient; Health personnel; Will. 


\section{INTRODUCCIÓN}

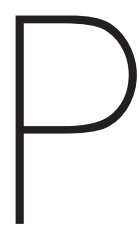

ara Talavera P. ${ }^{1}$ la Objeción de Conciencia (OC) es la negativa de un individuo a someterse, por razones morales, a una conducta que en principio le sería juńdicamente exigible, bien porque la obligación proviene de una ley general o bien porque se deriva de un contrato o de una resolución judicial o administrativa. Según Beca J. P. y Astete C.? la OC en el ámbito de la salud se ha definido como la negativa de los profesionales de la salud a cooperar o a ejecutar materialmente alguna intervención concreta que entra en colisión con sus imperativos de conciencia.

En cuanto a la Voluntad Anticipada (VA) Ávila J.A. ${ }^{3}$ sostiene que son el conjunto de preferencias que una persona tiene respecto del cuidado futuro de su salud, de su cuerpo y de su vida, y como ésta decide cuando está en pleno uso de sus facultades mentales, en anticipación, a la posibilidad de que en algún momento en el futuro, se encuentre incapacitada para expresar sus preferencias y tomar decisiones por sí misma.

Por lo anterior, la objeción surge cuando al querer cumplir un mandato, la conciencia se opone, por encontrar hechos contrarios a la moral o a los valores previos. Así, por ejemplo, si un acto médico es contrario a un valor moral, surge la negativa a llevarlos a cabo o de participar en ellos. De igual forma, la VA es la manifestación de voluntad de una persona con capacidad de ejercicio sobre su decisión de ser sometida o no a medios, tratamientos o procedimientos médicos que pretendan prolongar su vida cuando se encuentre en etapa terminal, y por razones médicas sea imposible mantenerla de manera natural, protegiendo en todo momento la dignidad de la persona.

\section{- Antecedentes de la Objeción de Conciencia}

Según Franca $\bigcirc .4$, la $\bigcirc C$ surgió por causas religiosas y militares. Como ejemplo, la respuesta del Apóstol Pedro -el primer papa- a las autoridades judías de Jerusalén de no acatar la prohibición de hablar públicamente de Jesucristo porque era su deber "obedecer a Dios antes que a los hombres". Así, Pedro pone su decisión por encima del derecho positivo. De igual forma, durante la segunda Guerra Mundial el presidente norteamericano Franklin D. Roosevelt aprobó que quienes rechazaran participar en servicios militares por causa religiosa o de conciencia, fuesen designados a trabajos civiles. Posteriormente, la ONU en la Declaración Universal de los Derechos Humanos, reconoció el derecho de toda persona a la libertad de pensamiento, de conciencia y de religión.

En el caso de México, de acuerdo a la Asamblea Legislativa del Distrito Federal ${ }^{5}$, sólo 11 estados tienen la Ley de Voluntad Anticipada en su legislación. Éstos son: Aguascalientes, Chihuahua, Coahuila, Colima, Estado de México, Guanajuato, Guerrero, Hidalgo, Michoacán, Nayarit y San Luis Potosí. En la Ciudad de México existe la Ley de Voluntad Anticipada desde el 7 de enero de 2008, reformada el 27 de agosto de 2012.

Entonces, la voluntad es una capacidad humana, necesaria para llevar a cabo todos los actos. Esto significa que es la expresión de necesidades y decisiones de una persona y para que sea válida jurídicamente debe ser expresada libremente por una persona mayor de edad y con pleno uso de sus facultades mentales. Es decir, sin influencia ni presión de ningún tipo por parte de otra persona. En caso de menores o personas incapaces, puede ser expresada por sus padres o un tutor legal.

- Valores éticos

Según De los Santos L. G. (6) el fundamento para la desobediencia al Derecho es la escisión entre la legalidad y la moralidad. Los motivos deben ser exclusivamente ético-morales y basados en la autonomía de la conciencia individual. Los conceptos de valor o moral, entendidos como preceptos personales basados en la libertad de creencias, son tomadas en cuenta, siempre que respete el bien común y no se lesionen los derechos de terceros. Así, las instituciones aunque no tienen

\section{6} MMN WMy Enf Neurol Vol.15. No. 2 mayo - agosto 2016 
conciencia moral, si presentaran una ideología, estatutos o códigos de ética que determinan su forma de trabajo. En el caso de instituciones de salud, son protocolos, que sin criterios normativos forman una responsabilidad moral que responde a criterios interdisciplinarios consensuados previamente.

- Aspectos legales de la Objeción de Conciencia

Según Martínez N. E.?, la libertad de pensamiento y de conciencia está protegida por convenios y pactos internacionales de derechos humanos, tanto en el ámbito global como en el nacional. En Europa por ejemplo, el Convenio Europeo de Derechos Humanos de 1950 en su artículo 9 declara que toda persona tiene derecho a la libertad de pensamiento, de conciencia y de religión. El artículo 2 de este Convenio, dice que a nadie se le puede negar el derecho a la instrucción y que el Estado respetará los derechos de los padres a la educación y la enseñanza conforme a sus convicciones religiosas y filosóficas.

Según Allier J. ${ }^{8}$ en el Sistema Interamericano, la Convención Americana sobre Derechos Humanos Pacto de San José, OEA- consagra la libertad religiosa, de pensamiento y de conciencia. México firmó también este pacto en 1980 y lo ratificó en 1981. La ONU en sus dos instrumentos sobre derechos humanos -el Pacto Internacional de Derechos Civiles y Políticos y el Pacto Internacional de Derechos Económicos, Sociales y Culturales- aprobados en 1996, también son vinculantes para México.

En México, únicamente Jalisco y la Ciudad de México han modificado la legislación para el reconocimiento de la OC como un derecho del personal del sistema de salud. La Ley Estatal de Salud del Estado de Jalisco reconoce este derecho desde el 7 de octubre de 2004. La Ciudad de México, reconoció la OC en 2004 y la ratificó en 2009. De hecho, la Secretaría de Salud aprobó las reformas a la NOM-046-SSA2-2005 el 27 de febrero de 2009 reconociendo el derecho a la $\bigcirc \mathrm{C}$ en médicos y enfermeras para la práctica del aborto. Por ello, la Ley de Voluntad Anticipada para el Distrito Federal, es un documento con validez legal, el cual, reconoce el derecho de la Objeción de Conciencia el personal de salud para abstenerse desde el 2008 de intervenir en la atención de pacientes terminales.

\section{LA TESIS: MUERTE DIGNA Y DERECHOS HUMANOS A LOS PACIENTES}

El final de la vida - la muerte-, para Méndez E.? depende de la manera en que se comprende el valor de la vida. Es una experiencia individual que está acorde a la visión personal de sí mismo (de la vida y la muerte digna) vinculadas con la enfermedad. Actualmente además con gran medicalización e institucionalización, la ciencia y la tecnología han desarrollado técnicas y métodos para curar y tratar enfermedades y la posibilidad de alargar la vida. Esto hace pensar que ha cambiado el paradigma en la atención a la enfermedad terminal y la manera en que las personas viven su fin.

Ahora, más que una muerte digna, médicos y enfermeras buscan proporcionar al individuo, una vida digna hasta el último momento, permitiendo que las personas vivan según su condición de seres humanos hasta su muerte, sin que alguien más tome las decisiones por ellos. Esto implica ejercer el respeto a la autonomía de la voluntad sobre el tipo de atención médica que el paciente desea recibir y hasta que momento desea recibirla.

En el caso de los Derechos humanos, Según Agulles P.10, estos son derechos que le corresponden al hombre como persona y son lógica y ontológicamente anteriores al Estado. Hacen referencia a las exigencias fundamentales de la persona, enraizadas en el mismo ser del hombre, que deben ser reconocidas, valoradas y defendidas jurídicamente. Su origen no está en la sociedad sino en el individuo mismo, como un ser-hombre.

Tratándose la $\bigcirc \mathrm{C}$ como un derecho fundamental y esencial de la persona humana, ningún Estado está en condiciones de otorgarlo ni derogarlo, pero sí tiene el deber de reconocerlo, protegerlo, tutelarlo y regularlo. Asimismo, está el derecho de

Enf Neurol Vol. 15. No. 2 mayo - agosto 2016 wh 
los enfermos a rechazar los tratamientos que prolonguen su vida, y obliguen a los familiares y a los médicos a respetar su decisión y salvaguardar en todo momento su autonomía, sus derechos humanos y su dignidad como persona enferma. Por ello, el documento de la VA otorga facilidad de ejercicio de la autonomía en el ámbito de los servicios de salud, dando al enfermo terminal la seguridad jurídica de que su decisión de una vida digna será respetada hasta el último momento. En estos casos, las Enfermeras y médicos deben también defender siempre este derecho de los pacientes a una muerte digna.

\section{- Objeción y conflictos}

Para Sierra B. S." no siempre es posible obtener la armonía entre los elementos personales de la relación jurídica médico-paciente y la posibilidad de una confrontación de derechos: el derecho a la protección de la salud, el derecho al libre ejercicio profesional y la autonomía del paciente. Así, el paciente puede llegar a objetar todos y cada uno de los tratamientos propuestos, ya sea por falta de confianza al personal médico o por sus propias convicciones. Por su parte, el médico puede también objetar las medidas demandadas por el paciente cuando el acto solicitado no sea acorde a su ética médica.

Lo anterior significa que una "objeción del paciente", permite plasmar su aceptación o rechazo al tratamiento otorgando o no su "consentimiento informado". Ahora, en el segundo caso, tratándose de la VA, surgen dilemas cuando el paciente ha expresado su derecho a rechazar un tratamiento terapéutico al final de su enfermedad, para únicamente recibir, cuidados paliativos.

Entonces, ¿se trata de una eutanasia pasiva, es decir, omisión de acciones médicas y terapéuticas, - de un suicidio asistido médicamente? Ambos supuestos están expresamente prohibidos en la legislación ${ }^{12}$. De aquí surge el término eutanasia que se deriva en ortotanasia cuando se da el cuidado evitando la utilización de tratamientos y/o procedimientos médicos, que al tornarse desproporcionados e inútiles, constituyen la distanasia.
- Toma de decisiones

La práctica médica y de enfermería se caracteriza por la toma de decisiones y la ejecución de acciones profesionales diversas basadas en una apreciación de aspectos científicos y técnicos y de los valores involucrados, Esto significa que, de manera anticipada, en el contexto de una enfermedad terminal, un paciente en estado de coma o en estado de sufrimiento irreversible, puede expresar su decisión cuando está en pleno uso de sus facultades mentales sobre cómo ha de disponerse de sus bienes más preciados: su salud, su cuerpo y su vida en el momento cercano a la muerte y en el que ya no sea capaz de decidir por sí mismo.

\section{CONCLUSIONES}

$\square$

a reflexión en torno a la vida y la muerte, son inherentes a la existencia de la persona humana y le han acompañado a lo largo de su historia; pero el tiempo ha ido cambiando la forma de conceptualizarlos debido a los conocimientos y a la posibilidad de intervenir mediante el uso de métodos y tecnologías cada más avanzados. De hecho, la interculturalidad y la multidiversidad de personas y tecnologías, también han determinado como han de ser éstos sucesos.

Ante éstas circunstancias, el ser humano tiene la necesidad de establecer normas y valores que le permitan elegir y establecer conductas que le lleven a un estado de bienestar individual y colectivo y que garantice su supervivencia, estableciendo aquellas que son comunes a todos y que se han llamado derechos humanos fundamentales. Aunque México es un Estado que ha reconocido plenamente los derechos fundamentales, protege los derechos humanos, se ha encontrado con un conflicto entre la Ley y la conciencia cuando se trata de los derechos de los pacientes y la obligación de los servicios de salud de velar por el bienestar de las personas.

Así, cuando en el ámbito clínico se tiene la posibilidad de alargar la vida o bien, prolongar la llegada de la muerte, surge la situación de mantener en todo

\section{8}

MMN $\mathrm{Wh}_{\mathrm{W}}$ Enf Neurol Vol. 15. No. 2 mayo - agosto 2016 
momento el respeto y la protección a los derechos y a la dignidad humana y por supuesto, el respeto a la autonomía de la voluntad del paciente. Para ello, se ideó que la voluntad de la persona pueda manifestarse de manera anticipada en un documento previo a su incapacidad, conteniendo las estipulaciones de una persona competente acerca de los tratamientos que desea o no recibir si llegase a presentar un estado de incapacidad.

Entonces, queda para el personal de salud, especialmente en los médicos y enfermeras la enorme responsabilidad de la reflexión sobre el respeto a la VA y la dignidad de la persona hasta el final de su vida, o la prevalencia de su conciencia moral y sus valores personales y profesionales.

\section{REFERENCIAS BIBLIOGRÁFICAS}

1. Talavera P. Objeción de conciencia y voluntades anticipadas. Facultad de Derecho. Universidad de Valencia. Madrid, 2010: 7-8. Disponible en http:// publicaciones.san.gva.es/cas/prof/bioetica/bioetica/docs/Prot.PedroTalaveraFernandez.pdf. Consultada 10 de septiembre de 2016

2. Beca I. J. P. y Astete A C. Objeción de conciencia en la práctica médica. Centro de Bioética. Facultad de Medicina. Clínica Alemana. Universidad del Desarrollo; Médica de Chile, Santiago de Chile, 2015; 143: 493-498.

3. Ávila J.A. ¿Qué es la voluntad anticipada? Instituto Nacional de Ciencias Médicas y Nutrición Salvador Zubirán. Ciudad de México, 2013: 1. Disponible en: http:/ / umw.innszmx/opencms/contenido/investigacion/comiteEtica/voluntadanticipada. html. Consultada 10 de septiembre de 2016

4. Franca O. La objeción de conciencia: Tres visiones sobre el tema. Prensa Médica Latinoamericana. Archivos de Medicina Interna. Uruguay, 2013; 35(2): $62-68$ Disponible en hittp:/ munv.scielo.edu.uy/scielo.php?script=sci_arttext HYPERLINK "hitto:/ muw.scielo.edu.uy/scielo.php?script=sci_anttext\&pid=S1688-423X2013000200007"\&

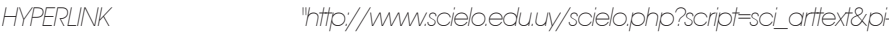
$d=S 1688-423 \times 2013000200007 " p i d=S 1688-423 \times 2013000200007$. Consultada el 2 de octubre de 2016.

5. Asamblea Legislativa del Distrito Federal. Ley de Voluntad Anticipada para el Distrito Federal. Instituto de Investigación Parlamentaria. Asamblea Legislativa VI Legislatura. México, 2012: 1-12. Disponible en http://www.aldf. gob.mx/archivo-edfcbf4442.b58c1cf761114a6a224fb1.pdf. Consultada el 25 de octubre de 2016.

6. De los Santos L. G. La objeción de conciencia: Fundamentos y justificación Centro de Bioética Juan Pablo Il. Septiembre - Diciembre. Habana de Cuba, 2010; 10(3): 1-4. Disponible en http://muw.cbioetica.org/suplement/103_sup. pdf. Consultada el 20 de septiembre de 2016
7. Martínez N. E. Artículo 10-BIS a la Ley General de Salud. Sistema de Información Legislativa de la Secretaría de Gobernación. Grupo Parlamentario del PES Ciudad de México, 2015: 7-5. Disponible en http://sil.gobernacion.gob.mx/ Archivos/Documentos/2015/11/asun_3295635_20151104_1446657855.pdt. Consultada el 25 de octubre de 2016

8. Allier J. Improcedencia de la objeción de conciencia planteada por un juzgador. Instituto de la Judicatura Federal. Enero, Ciudad México 2012: 33: 11 - 21 Disponible en: http://www.ijf.cjf.gob.mx/publicaciones/revista/33/02\%20JAME\%20ALLIER\%20CAMPUZANO.pdf. Consultada el 10 de septiembre de 2016

9. Mendoza F. Voluntades anticipadas: reflexiones bioéticas sobre el final de la vida. Conbioética s/n. Secretaría de Salud. México, 2015: 1-11. Disponible en: conbioetica-mexico.sa/ud.go.b.mx/descargas/pdf/voluntades_anticipadas.pdf. Consultada el 10 de septiembre de 2016.

10. Agulles P. S. La objeción de conciencia farmacéutica en España. Edizioni Universidad Santa Croce, Roma 2006: 1 - 519. Disponible en http://bibliotecanonica.net/docsal/btcalx.pdf. Consultada el 12 de septiembre de 2016.

11.Sierra B. S. Objeción de conciencia en el ámbito médico-sanitario en México. Jurídica. Universidad Latina de América. Septiembre - Diciembre. México, 2017: 132: 1-6. Disponible en file:///C:/Users/HP/Down/oads/47106072-1-PB.pdf Consultada el 10 de septiembre de 2016.

12. Asamblea Legislativa del Distrito Federal. Código Penal para el Distrito Federal, Artículos 143 bis y 158 bis. México, 2016:33, 35. Disponible en: http:// aldf.gob.mx. Consultado el día 1 de mayo del 2016 